\title{
Experimental and Numerical Investigation of the Horizontal Ribbon Growth Process
}

\author{
Brian T. Helenbrook ${ }^{\mathrm{a}, *}$ Peter Kellerman ${ }^{\mathrm{b}}$ Frederick Carlson ${ }^{\mathrm{a}}$ \\ Nandish Desai ${ }^{b}$ Dawei Sun ${ }^{b}$ \\ ${ }^{a}$ Department of Mechanical and Aerospace Engineering \\ Clarkson University, Potsdam, NY, 13699-5725, USA \\ ${ }^{\mathrm{b}}$ Varian Semiconductor Equipment Division \\ Applied Materials, Inc., Gloucester, MA, 01930, USA
}

\begin{abstract}
Experimental and numerical results are presented on the process of horizontal ribbon growth (HRG) of single-crystal silicon. Experimental data on the leading edge position of the growth front as a function of pull speed is compared to model predictions with and without solidification kinetic effects. Without kinetics, the numerical results predict leading edge positions which are completely different than that observed in the experiment. With kinetics, the leading edge position is predicted typically within $1 \mathrm{~mm}$ and the change in position with pull speed also is well predicted. Conclusions from the kinetic model are that the growth occurs through a faceted process where the leading edge is a $\{111\}$ facet that requires significant supercooling to maintain the growth. An outcome of the model is that the leading edge position versus pull speed response shows a turning point beyond which there are no steady growth solutions. This is consistent with all previously reported experiments on this process, which have reported maximum attainable pull-speeds. These results
\end{abstract}

Preprint submitted to the Journal of Crystal Growth

(C) 2016. This manuscript version is made available under the Elsevier user license http://www.elsevier.com/open-access/userlicense/1.0/ 
directly contradict previous conclusions from the "wedge" model of horizontal ribbon growth, which predicts that a large area wedge-shaped growth region exists and that increasing pull speeds simply result in a narrower wedge angle.

Key words: A1 solidification, A1 heat transfer, A2 growth from melt, A1 growth models, A2 horizontal ribbon growth

\section{Introduction}

Horizontal ribbon growth (HRG) is a technique for producing thin solid sheets of a material (typically silicon). Heat is removed from the free surface of a molten pool, which causes the top surface to solidify. This solid is then pulled horizontally with a specified velocity, $u_{s}$, such that a steady-state production process is established. There are different types of horizontal ribbon growth processes (see [1 4] for overviews) such as horizontal supported web (HSW)

\footnotetext{
* Corresponding author
}

Email address: helenbrk@clarkson.edu (Brian T. Helenbrook). 
[5], ribbon growth on substrate (RGS) [6], and methods in which there is no contact with a substrate [7, 8]. These last methods are often just called horizontal ribbon growth or in some cases the low-angle silicon sheet method (LASS) 9]). It is these methods that are of interest in this paper, and in the following the term horizontal ribbon growth is meant to refer specifically to these methods.

There have been various HRG experiments that differ in their approaches to seeding the growth and the types of crystals grown. Kudo [10] reported growing ribbons with maximum growth rates of $415 \mathrm{~mm} / \mathrm{min}$ for single crystal sheets and $850 \mathrm{~mm} / \mathrm{min}$ for multi-crystalline sheets. Similarly, Bates and Jewett [9] reported growing multi-crystalline ribbons at pull speeds of $850 \mathrm{~mm} / \mathrm{min}$ and also briefly mentioned that a limited amount of crystals were grown with a "planar front" at a pull speed of $300 \mathrm{~mm} / \mathrm{min}$ which is close to the maximum pull speed reported by Kudo for single-crystal sheets. Cheng et al. [11, 12] grew bi-crystalline ribbons where there was a twin plane along the center of the seed and reported growing primarily single crystal material at pull speeds of 10-30 mm/min "with plenty of room for improvement."

To predict these relatively large growth rates compared to Czochralski growth or vertical growth methods, most analyses of HRG [10, 13 16] use simplified energy considerations. For example, given a free-surface heat removal rate, a solidification velocity can be estimated. The ratio of this solidification velocity to the speed at which the solid is removed from the device determines an angle of growth for the solidifying wedge. Based on this, it was predicted that the same thickness sheet could be produced at higher speeds simply by increasing the area of growth (decreasing the wedge angle) and that there is no intrinsic limit on the pull speed except for the length of the resulting wedge in the 
experimental apparatus. Further refinements of the wedge analysis assuming that the leading edge shape was parabolic [17] also came to similar conclusions.

More recently, numerical simulations of the Stefan problem have been used to verify the conclusions of the wedge analysis [18 20]. In [18], it was shown that the growth wedge changes length as a function of pull speed, and it was also shown that, depending on the assumed boundary conditions, supercooling could appear in front of the solidification front. Ranjan et al. [19] found wedgetype behavior in their simulations using a phase-field approach and concluded, "It is therefore possible to achieve a high production rate since the process rate has been decoupled from the rate limitation imposed by crystallization." Daggolu et al. [20] came to similar conclusions and showed that modifying the geometry to allow a longer wedge with the same heat removal rate enables higher pull rates while maintaining sheet thickness. More recent studies have examined additional limiting factors such as meniscus stability effects (where molten material spills from the crucible) and bridging effects (where the solid freezes to the crucible) [21, 22] as well as segregation effects [23, 24].

In our recent experimental studies, a new horizontal ribbon growth apparatus was employed where the growth region is isolated from the extraction region as shown in figure 1. In the region between the growth and extraction region, the solid floats on the molten liquid. Thus this approach is only applicable to materials whose solid phase is less dense than its liquid phase (such as silicon). For this reason, we call this approach the floating silicon method (FSM). The advantage of FSM is that it separates the growth process from the extraction process making both easier to control and study. In the following, results are reported on the growth of single-crystal silicon using the FSM process. As discussed in this paper, experimental results obtained by FSM do not agree 
with previous wedge based theories; for a given heat removal rate, there is a limitation to the pull speed which is not related to the experimental geometry. The experiments show that as the pull speed is increased, the position of the leading edge of the growth interface retreats until at some pull-speed a stable pulling process cannot be established. To explain this, numerical simulations were performed that show that this behavior can only be predicted by including finite rate solidification kinetics and faceted growth in the model. The simulation results with kinetics show that the single-crystal growth mechanism for this process is not a wedge at all but rather a faceted growth process.

\section{Experimental Configuration}

A schematic of the FSM configuration used for the experiments is given in figure 1. Shown in the figure is a molten pool of silicon, a quartz crucible, and a graphite support structure. The support structure was surrounded by heaters in an enclosed furnace to allow temperature control of the environment. These heaters were adjusted to maintain a nearly isothermal environment near the melting temperature of silicon. Conceptually, the length of the furnace can be divided into 5 regions: a replenishment region, a growth region, a thickness control region, a separation region, and finally a removal and cutting region. The region of interest is the growth region. The quartz crucible was long enough to make the physics of each region somewhat independent. As mentioned in the introduction this allowed the growth physics to be studied independent of separation physics such as meniscus stability and bridging effects.

For the experiments reported on in this paper, a heat removal device was 
constructed whose purpose was to allow optical access to the growth process while also being easy to thermally model. This device consisted of a helium jet that emanated from a slot in a horizontal planar surface, which we call the "cold block", and impinged on the top surface of the melt. The slot was narrow and uniform and thus created a predominately 2D heat removal profile that sustained the solidification process. To allow optical access from the side of the furnace, silicon sheets grown with this device were limited to a width of less than $4 \mathrm{~cm}$.

Figure 2 shows the top surface of a sheet pulled using the FSM apparatus. This sheet was grown with a different heat removal device that allowed wider sheets to be produced, but the basic characteristics of the sheets grown are similar. To initiate the solidification process a silicon seed was inserted into the apparatus, floated on the melt surface, and then pulled from the device as the helium jet was turned on. The initial seed (visible on the right side of the figure) was $2 \mathrm{~cm}$ wide. The seed was a single crystal sheet of silicon made from an electronics grade silicon wafer oriented such that the (100) face was on top and the pull direction was the $[0 \overline{11}]$ direction. The seed was connected to a puller that was used to extract the seed at a constant rate. As the seed grew, it widened to a width determined by the heat removal device. In the image shown the sheet width was $14 \mathrm{~cm}$. The horizontal line down the middle of the top surface was due to non-uniformity in the heat removal profile. The simpler heat removal device used for the experiments did not create any noticeable transverse irregularities and created sheets of about $4 \mathrm{~cm}$ wide. During the experiments a video camera was used to record the location of the growth front relative to the center of the helium jet as a function of pull speed. This image was corrected for parallax errors and then used to determine the position of the 
solidification front as a function of the pull speed. A calibration measurement was done with a ruled surface to validate the method of correcting for parallax errors.

During the growth process several observations were made that indicated that the crystallography of silicon plays a strong role in this process. In early experiments, the sheet was pulled slowly from the device after the cooling was shut off. When these seeds were removed, the leading edge of the sheet was almost entirely planar making an angle of $55^{\circ}$ from the top surface. An image is shown in figure 3. This is approximately the angle that would be formed by a $\{111\}$ facet. Another indication that the kinetics play a strong role in the growth process was that in the video images the solidification front was nearly always perfectly straight. These observations led us to question the wedge model of growth and postulate that a faceted growth process was more likely. Further evidence confirming this fact is given in a companion paper that reports more of the experimentally observed details [25]. Previous authors have discussed faceting effects for silicon grown on free-surfaces [12, 26], but they did not relate this to the limits of pull speed for a horizontal ribbon growth process.

\section{Solidification Modeling}

The solidification model consisted of a solid and liquid separated by a solidification interface as shown in figure 4, which also shows a typical triangular mesh used for the calculations. The domain, $\Omega$, is subdivided into two subdomains, $\Omega_{s}$ and $\Omega_{l}$, where in all of the following subscripts of $s$ and $l$ denote solid and liquid respectively. The governing equation in the solid is the convection- 
diffusion equation

$$
\frac{\partial \rho_{s} c_{s} T}{\partial t}+\frac{\partial \rho_{s} u_{s, j} c_{s} T}{\partial x_{j}}+\frac{\partial}{\partial x_{j}}\left(-k_{s} \frac{\partial T}{\partial x_{j}}\right)=0
$$

where $t$ and $x_{j}$ are the temporal and spatial coordinates. Indicial notation is used and the problem is $2 \mathrm{D}$ so $j \in[1,2] . \rho$ is the density, $c$ is the specific heat, $k$ is the thermal conductivity, $T$ is the temperature, and $u_{s, 1}$ is a specified rigid body velocity for the solid. The solid was pulled horizontally so $u_{s, 2}=0$. For the solidification calculations all properties were assumed to be constant and $\rho_{s}$ was assumed to be equal to $\rho_{l}$. The specific values used for the following calculations were $\rho_{s}=2530 \mathrm{~kg} / \mathrm{m}^{3}, c_{s}=1000 \mathrm{j} /(\mathrm{kgK})$, and $k_{s}=22 \mathrm{~W} /(\mathrm{mK})$ [27].

The governing equations in the liquid domain again include the heat equation except the flow velocity $u_{l, j}$ is determined by the Navier-Stokes equations

$$
\begin{aligned}
& \frac{\partial \rho_{l} u_{l, i}}{\partial t}+\frac{\partial \rho_{l} u_{l, j} u_{l, i}}{\partial x_{j}}=-\frac{\partial p}{\partial x_{i}}+\frac{\partial \tau_{i, j}}{\partial x_{j}} \\
& \frac{\partial u_{l, j}}{\partial x_{j}}=0
\end{aligned}
$$

where $p$ is the fluid pressure and $\tau_{i, j}$ are the viscous stresses. The viscous stresses were calculated using a Newtonian relationship, $\tau_{i, j}=\mu\left(\frac{\partial u_{l, j}}{\partial x_{i}}+\frac{\partial u_{l, i}}{\partial x_{j}}\right)$ where $\mu$ is the liquid viscosity. For the liquid, constant properties were again assumed with $\rho_{l}=\rho_{s}, c_{l}=c_{s}, k_{l}=64 \mathrm{~W} /(\mathrm{mK})$, and $\mu=7 \times 10^{-4} \mathrm{~kg} /(\mathrm{ms})$ [27].

The liquid and solid domains are separated by the solidification interface, $\Gamma_{I}$. Mass conservation across the interface gives

$$
\llbracket \rho\left(u_{j}-x_{j, t}\right) n_{j} \rrbracket_{\Gamma_{I}}=0
$$


where the double brackets indicate the jump in the quantity across the interface and the subscript with respect to $t$ indicates a time derivative. $x_{j, t}$ is thus the interface velocity. $n$ is an outward normal; the change in sign for the jump is included by the opposite directions of the normal as defined from the solid and liquid domains. For this work, because the solid and liquid densities are assumed equal there is no jump in normal velocity across the interface. As there is no jump in tangential velocity either, the velocity is continuous across the interface. Furthermore, because the solid velocity is specified, the interface is a Dirichlet boundary condition for the flow where the flow velocities are set equal to the solid velocity.

Energy conservation across the interface gives

$$
\left[\left[\left(\rho c T\left(u_{j}-x_{j, t}\right)-k \frac{\partial T}{\partial x_{j}}\right) n_{j}\right]\right]_{\Gamma_{I}}=\rho_{s}\left(u_{s, j}-x_{j, t}\right) n_{s, j} L_{f}
$$

which says that the net energy flux to the interface is responsible for the conversion of solid to liquid with $L_{f}$ being the latent heat of fusion which was taken as $1.8 \times 10^{6} \mathrm{j} / \mathrm{kg}[27]$.

Solidification kinetics are included in the model using the model proposed by Weinstein and Brandon [28]. This model gives a relationship between the interface temperature and the growth velocity of the solid as

$$
\Delta T=K(\theta, \Delta T)\left(u_{s, j}-x_{j, t}\right) n_{s, j}
$$

where $\Delta T=T_{m}-T$ evaluated on the interface and $T_{m}$ is the equilibrium melting temperature which was assumed to be $1685 \mathrm{~K}$. The right hand side is a kinetic constant times the solidification velocity. The form used here is slightly different than that presented in [28] where the relationship was written 
as $v_{g}=\beta \Delta T$ where $v_{g}$ is the solidification velocity and $\beta=1 / K$. The above form allows one to simulate the Stefan problem by setting $K=0$.

The kinetic constant is a composite term that models three mechanisms of solidification: roughened growth, step propagation, and 2D nucleation. Roughened growth is modeled with a constant kinetic coefficient as $\Delta T=K_{\text {rough }} v_{g}$. Growth of the solid by propagation of molecular steps across a low-index surface such as a $\{111\}$ facet is modeled by $\Delta T=\frac{K_{\text {step }}}{|\sin (\theta)|} v_{g}$ where $K_{\text {step }}$ is a constant and $\theta$, the misalignment angle, is the angle between the solidification interface normal and the facet normal. Growth by the formation of clusters of atoms on a low-index surface is modeled by a 2D nucleation kinetic constant which is given by $\Delta T=B^{-1} \exp (A / \Delta T) v_{g}$ where $A$ and $B$ are constants. These expressions are explained in more detail in [28]. The three mechanisms are combined together using the following expression

$$
K=\left(K_{\text {rough }}^{p}+K_{\text {min }}^{p}\right)^{1 / p}
$$

where $p$ can be any integer, but for the following simulations a value of 2 was chosen. The above essentially choses the maximum value between $K_{\text {rough }}$ and $K_{\text {min }}$ ensuring that the amount of supercooling never goes below that needed for roughened growth. $K_{\min }$ is given by

$$
K_{\text {min }}=\min \left(K_{2 D N}, \frac{K_{\text {step }}}{|\sin (\theta)|+\epsilon_{\text {step }}}\right)
$$

This expression chooses the process that requires less supercooling to define $K_{\min } . K_{2 D N}$ is given by

$$
K_{2 D N}=B^{-1} \exp \left(\frac{A}{\max \left(\Delta T, \epsilon_{2 D N}\right)}\right)
$$


The values of $K_{\text {rough }}, K_{\text {step }}, A$, and $B$ were obtained from [28]. $K_{\text {rough }}=$ $79.4 \mathrm{Ks} / \mathrm{m}, K_{\text {step }}=1.59 \mathrm{Ks} / \mathrm{m}, A=140 \mathrm{~K}$, and $B=1.5 \times 10^{10} \mathrm{~m} /(\mathrm{sK})$. $\epsilon_{\text {step }}$ and $\epsilon_{2 D N}$ are numerical constants introduced to avoid division by zero errors. $\epsilon_{\text {step }}$ was set to machine epsilon $\approx 1 \times 10^{-16} . \epsilon_{2 D N}$ varied depending on the simulation. For initial transients, $\epsilon_{2 D N}$ was set to $4 K$, but for final steady solutions, $\epsilon_{2 D N}$ was set to $1 \mathrm{~K}$. In the following calculations, when $\epsilon_{2 D N}$ was set to $1 K$, it no longer affected the solution, because in all the regions where $K_{\min }$ was determined by $K_{2 D N}$, the supercooling, $\Delta T$, was greater than $1 K$. Another adjustment that was made during initial transients was that $K_{\text {step }}$ was increased by a factor of 90 . As $K_{\text {step }}$ decreases, the range of misalignment angles that causes $K_{\text {rough }}<K_{\text {step }} /|\sin (\theta)|<K_{2 D N}$ decreases. This then requires a highly accurate prediction of the interface position to capture the faceting behavior. By increasing $K_{\text {step }}$ during initial transients, we were able to more easily resolve the facet dynamics and reach a solution that could be further refined by decreasing $K_{\text {step }}$ to the physically correct value.

\subsection{Boundary and Initial Conditions}

In the solidification modeling, only the growth region of the experimental configuration is modeled. The domain is shown in figure 4 where the units are scaled by the depth of the molten pool, $d$.

\subsubsection{Flow Boundary Conditions}

On the left side of the domain shown in figure 4 , an inflow boundary condition is used to model the flow of molten silicon from the replenishment region. On 
this boundary, the velocity was constrained to be an open channel flow profile given by

$$
u_{1}=u_{s, 1}\left(1-\left(x_{2} / d\right)^{2}\right)
$$

where $u_{s, 1}$ is the horizontal pull speed of the solid. This provided more than enough flow to compensate for the rate of solid material removed from the domain.

In order to enable a steady-state solution to be obtained, an outflow boundary condition was used for the liquid on the right side of the domain where the total stress on the boundary, $\left(-p \delta_{i, j}+\tau_{i, j}\right) n_{j}$ where $\left(n_{1}, n_{2}\right)=(1,0)$, was set to zero. This allowed any surplus replenishment flow to exit the domain. In actuality, a surplus replenishment flow would cause the liquid level to rise, but that dynamic was not included in the solidification model.

The top surface of the liquid was treated as a slip boundary condition. For all of the conditions studied here, the flow velocity was such that the Weber number, $W e=\rho_{l} u_{1}^{2} d / \sigma$ where $\sigma$ is the surface tension of silicon was less than 0.1. Similarly, the Froude number, $u_{1} / \sqrt{g d}$, was also less than 0.1 so approximating the free-surface of the silicon as a flat slip surface was an accurate approximation. However, an effect that is significant is the Marangoni stress. This was included by applying a shear stress to the top surface given by

$$
\tau_{1,2}=\frac{d \sigma}{d x_{1}}=\frac{d \sigma}{d T} \frac{\partial T}{\partial x_{1}}
$$

When this effect was included, the value used for $\frac{d \sigma}{d T}$ was $-7 \times 10^{-5} \mathrm{~N} /(\mathrm{mK})$ [27]. 
At the bottom surface of the liquid a no slip wall boundary condition was applied where both components of the velocity were set to zero.

\subsubsection{Thermal Boundary Conditions}

The top surface heat flux caused by the helium jet were estimated using simulations performed in ANSYS ${ }^{\circledR}$ Fluent, Release 16.2 for three different heat removal configurations. Heat flux results are shown in figure 5. The inset of the figure shows typical streamlines obtained from the ANSYS simulations of the jet impingement problem. The dashed curves in the figure are curve-fits which were created to allow the heat flux profiles to be easily incorporated in the solidification simulations. The form for the curve fit was a combination of two Gaussians and a constant base flux as given by

$$
q_{c}=q_{\text {peak }}\left[(1-\theta) e^{-(x / w)^{2}}+\theta e^{-\left(x / w_{b}\right)^{2}}\right]+q_{\text {base }}
$$

where $q_{\text {base }}, q_{\text {peak }}, w, w_{b}$, and $\theta$ are curve fit parameters. $q_{b a s e}$ is the base heat flux due to conduction (no helium flowing). $q_{\text {peak }}$ is the increase in heat flux caused by the jet. The parameters $w$ and $w_{b}$ are length scales. $w$ primarily scales with the width of the slot while $w_{b}$ scales with the height between the slot exit and the silicon surface. $\theta$ controls the transition between the narrow peak (width $w$ ) and the wider base profile (width $w_{b}$ ). For the three curves shown on the figure, the curve fit parameters are shown in table 1 . These are the cases for which comparisons to experimental data were made and are shown in order of increasing peak heat flux at $x_{1}=0$.

The jet heat flux profile was modified to include radiation effects and to account for the fact that the planar surface from which the jet emanated had a 
finite streamwise span. This surface was the bottom of a cold block of width $5 \mathrm{~cm}$ with the slot located at the midpoint. To model the radiation exchange, surrounding radiative surfaces other than the bottom of the block were assumed to be close to $T_{m}$ so that the dominant radiation transfer could be assumed to be between the free surface and this surface. The emissivity values modeled silicon with the liquid emissivity, $\epsilon_{l}$, equal to 0.2 and the solid emissivity, $\epsilon_{s}$, equal to 0.6 [27]. Furthermore, it was assumed that temperature variations along the free surface were small relative to $T_{m}$. In this case, the black-body-radiation heat flux function can be approximated as

$$
q_{r}=\epsilon \sigma_{b} F\left(x_{1}\right)\left(T_{m}^{4}-T_{c}^{4}\right)
$$

where $\sigma_{b}$ is the Stefan-Boltzmann constant, the appropriate value of $\epsilon$ is chosen depending on whether $q_{r}$ is evaluated on the solid or liquid top surface, and $T_{c}$ was assumed to be $300 K . F\left(x_{1}\right)$ is a view factor (see [29] B-71) which is nearly 1 under the block and goes to 0 beyond the edges of the block as defined by

$$
F\left(x_{1}\right)=\frac{\sin \left(\phi_{2}\right)-\sin \left(\phi_{1}\right)}{2}
$$

where $\sin \left(\phi_{1}\right)$ and $\sin \left(\phi_{2}\right)$ are given by

$$
\begin{aligned}
& \sin \left(\phi_{1}\right)=\frac{-w_{r}-x_{1}}{\sqrt{\left(-w_{r}-x_{1}\right)^{2}+h_{\text {slot }}^{2}}} \\
& \sin \left(\phi_{2}\right)=\frac{w_{r}-x_{1}}{\sqrt{\left(w_{r}-x_{1}\right)^{2}+h_{\text {slot }}^{2}}}
\end{aligned}
$$

and $w_{r}$ is the half width of the block. Because of the jump in emissivity, the radiative heat flux is not continuous at triple junction. We have shown previously that for the Stefan problem the jump in heat flux at the triple 
junction determines the angle at which the solidification front meets the top surface [30]. As we will see in the results below however, this is no longer true when kinetics are included.

The radiative flux is added to the convective heat flux profile determined for the helium jet. There was one slight modification to account for the finite length of the cold block. The term $q_{b a s e}$ in eq. 6, which models the conduction between the silicon and the cold block, was multiplied by the view factor function $F\left(x_{1}\right)$ so that the conductive flux went to zero beyond the edges of the cold block. For the conditions listed in table 1, the radiative flux was at the most ten percent of the total heat removal, with a peak value of $27 \mathrm{~W} / \mathrm{cm}^{2}$.

A heat flux profile was also specified along the bottom surface of the domain. Most of the heaters under the experiment were set to maintain an isothermal environment, however there was one heater located under the cold block that provided a net positive flux during the growth process. This was done to maintain a positive thermal gradient in the silicon, which was thought to provide a more stable growth environment. The width of this heater was the same as the width of the cold block. Thermal conduction simulations of the entire furnace were performed to determine the heat flux provided to the liquid silicon as a function of the power supplied to the heater. Figure 6 shows the thermal heat flux caused by this heater when the input was $900 \mathrm{~W}$ as calculated by the full furnace Fluent model. The figure also shows a curve fit defined by $q_{b}=\frac{P}{900 W} 22.25 \mathrm{~W} / \mathrm{cm}^{2} F\left(x_{1}\right)$ where $P$ is the input power to the heater in Watts and for convenience we have used the view factor function to model the drop off in heat flux at the edges of the heater. For the three jet conditions given in table 1 , the heater powers were 1000, 1250, and $1000 \mathrm{~W}$ respectively. 
To promote stable thermal conditions upstream and downstream in the domain an additional "pass through" heat flux term of the form $q_{\text {pass }}\left(1-F\left(x_{1}\right)\right)$ was removed from the top and added to the bottom heat flux removal profiles. This term only has an effect outside of the growth region because of the $1-F\left(x_{1}\right)$ term. Because the same amount of heat is removed and added, this term provides no net impetus for growth. The heaters in the experiment were set-up so that there was little growth or melting except under the cold block, but the net flow of heat from the bottom to the top of the furnace was not measured. In the following calculations, a value of $2 \times 10^{4} \mathrm{~W} / \mathrm{m}^{2}$ was used for $q_{\text {pass }}$. This is a negligible flux compared to the fluxes under the cold block but was found to be necessary to avoid thermal instabilities downstream of the growth region for the simulations without kinetics given in the results section.

At the inflow, the temperature was given by

$$
T=T_{\text {inlet }}-\frac{q_{\text {pass }} x_{2}}{k_{l}}
$$

where $T_{\text {inlet }}$ was $5 K$ above the melting temperature of silicon and the linear term is added to be consistent with the stabilizing pass through heat flux $q_{\text {pass }}$. The validity of this condition relevant to the experiment is difficult to assess, as there was no way to directly measure the temperature at this location. The value of $5 \mathrm{~K}$ was chosen because in the initial set-up of the experiment, the heaters were adjusted such that a silicon seed inserted into the furnace (before the helium jet was turned on) melted back slowly. This indicates a temperature slightly above melting temperature.

At the outflow of the domain, a zero heat flux condition was enforced for both the solid and liquid, $\frac{\partial T}{\partial x_{1}}=0$. In the thickness control region of the experi- 
ment, the heater settings and geometry were nearly uniform in the horizontal direction so this should be an accurate approximation.

\subsubsection{Initial Conditions}

The solid shape shown in figure 4 was used to initialize the calculations, which were then run until a steady-state solution was obtained. This shape is given by

$$
x_{2}=-t_{\text {seed }}\left(1-\exp \left(-x_{1} \tan \left(54.7^{\circ}\right) / t_{\text {seed }}\right)\right)
$$

where $t_{\text {seed }}$ is the initial seed thickness, which was chosen as $0.2 d$. This shape was chosen because as it approaches the top surface the slope approaches that

of a (111) facet. To initialize the temperature and flow field, a steady-state thermal-fluid calculation was performed with the interface position fixed and the interface temperature held at $T_{m}$. The initial pull velocity was set to a small value, typically on the order of $.4 \mathrm{~mm} / \mathrm{s}$.

\subsection{Numerical Method}

The above equations were solved using the high-order-accurate numerical method described in [31]. In this method, the solidification front is tracked using a triangular, arbitrary-Lagrangian-Eulerian (ALE) moving mesh, and an $h p$-finite element method is used to calculate the thermal and flow fields. This is combined with an A-stable diagonally implicit Runge-Kutta temporal scheme and a mesh adaption algorithm that maintained mesh quality as the mesh deformed and also resolved sharp gradients such as those that occur near the triple junction. The method was demonstrated to give a temporal order of 
accuracy near 3 by comparing to a 1D analytic solution of melting. The spatial accuracy was calculated to be nearly $5^{\text {th }}$ order when using a polynomial approximation space of degree 4, which is what was used in all of the following calculations. Additional validation calculations for this algorithm can also be found in [30].

The transient simulations were run with an adaptive time step and adaptive mesh. As the transient simulations were only performed with the goal of converging to steady solutions, these calculations were run with first order polynomials and the mesh adaptation scheme was set to maintain the mesh quality and initial mesh density as the mesh deformed. It was not used to adjust the mesh resolution during the transient. The initial time step was $\alpha_{l} \Delta t / d^{2}=1 \times 10^{-4}$ where $\alpha_{l}$ is the thermal diffusivity of the liquid. Throughout the calculation the time step increased until a steady calculation could be performed. After a steady-solution was obtained, $\epsilon_{2 D N}$ was set to $1 K$, the polynomial order was increased to 4 , and the mesh adaptation scheme was allowed to adjust the mesh density to better resolve sharp gradients in the solution. Using this well resolved steady solution as a starting point, a quasisteady evolution was calculated where steady solutions were obtained with increasing pull-speeds. At each pull speed, Marangoni effects were then added and $K_{\text {step }}$ was decreased to the physical value.

\section{Simulation Results}

Figure 7 shows temperature contours and the interface position calculated for the third case given in table 1 at pull speeds of $1.2,1.6$, and $2.2 \mathrm{~mm} / \mathrm{s}$. There are several features of these plots to be examined. From a high-level 
perspective, the triple junction stabilizes slightly upstream of $x_{1}=0$ which is the center of the helium jet. Most of the growth occurs around $x_{1}=0$ area with some additional thickening caused by the cold block, which terminates at $x_{1}=2.5 \mathrm{~cm}$. Beyond this point, the interface then becomes nearly completely horizontal indicating that there is no solidification occurring.

Comparing the three different pull speeds shows that the thickness of the sheet decreases with pull speed. This is expected based on energy conservation; the total heat removed from the domain should be equal to the energy released by solidification, $Q_{n e t}=\rho L_{f} u_{s, 1} t_{\text {sheet }}$ where $t_{\text {sheet }}$ is the thickness of the sheet leaving the domain and $Q_{n e t}$ is the total heat removal from the domain. The total heat removal is mainly determined by the jet and is almost independent of pull speed. There is some dependence because the convective fluxes at the inlet at outlet change with pull speed. The radiation energy flux also has a dependence on pull speed because the position of the triple junction can shift, changing the emissivity of the top surface. However, both of these effects are small, and therefore the sheet thickness should be inversely proportional to pull speed. This is confirmed in figure 8 which shows thickness versus pull speed as well as a curve of the form $t_{\text {sheet }}=C / u_{s, 1}$ as a dashed line.

The temperature contour plots also show that at the leading edge of the sheet there is a $\{111\}$ facet. This can be seen in the blow-up region at each pull speed; starting at the triple junction there is a nearly flat facet at an angle of $55^{\circ}$ clockwise from the horizontal. Thus this model predicts that the primary growth of the sheet is by faceted edge growth and not wedge growth. At some depth the facet transitions away from a $55^{\circ}$ slope and the solidification front smoothly curves towards horizontal. In the following, this location is called the "elbow". The temperature contours in the blow up region show that the 
$T_{m}-5 \mathrm{~K}$ contour terminates very near the triple junction and the $T_{m}-2.5 \mathrm{~K}$ contour intersects the facet. The $T_{m}$ contour cannot be seen in the blowup region. This contour, which can be seen in the large-scale image, seems to intersect the interface at the transition where the interface turns towards horizontal. In reality, the $T_{m}$ contour does not intersect the interface at this point but sharply bends to the right to closely parallel the interface. This is consistent with the fact that where the solidification rate is small the interface temperature should be close to $T_{m}$.

At the triple junction, where the interface is the most super-cooled, the kinetic expression is determined by the $2 \mathrm{D}$ nucleation expression. Between the triple junction and the elbow, the kinetic expression is determined by the step propagation expression, and beyond the elbow, the roughened growth constant determines $K$. Figure 9 shows the misalignment angle as a function of horizontal position in a $2 \mathrm{~mm}$ region starting at the triple junction. The inset shows the shape of the interface with the labels $\mathrm{A}, \mathrm{B}$, and $\mathrm{C}$ in both plots corresponding to the triple junction, the beginning of the elbow, and the end of the elbow respectively. Along the facet, the misalignment angles are small but increase towards the elbow. The beginning of the elbow occurs where $K_{\text {step }} /|\sin (\theta)|=K_{\text {rough }}$ which is an angle of $1.15^{\circ}$. This agrees well with the plot, where at point $\mathrm{B}$ a sharp change in misalignment angle occurs from around $1.5^{\circ}$ to nearly $20^{\circ}$ at point $\mathrm{C}$ which is roughly the end of the elbow. After that the misalignment smoothly approaches $55^{\circ}$ which corresponds to a horizontal interface. Thus, the model predicts a growth and spread picture of the solidification; 2D Nucleation occurs at the triple junction creating steps that then propagate down the facet, slowing at the elbow such that the misalignment angle increases to the point that roughened growth takes over and 
the interface continuously bends towards horizontal. Our more recent experimental data [25] indicate that this is an oversimplified view of the process especially very near the triple junction (point A), however as we show below, this kinetic model does well-predict the response of the system to changes in pull-speed.

Another observation from the contour plots is that there is a significant amount of liquid that is supercooled. For the three pull speeds shown, the $T_{m}$ contour continues from the interface to around $2 \mathrm{~cm}$ upstream, which is near the leading edge of the cold block. The minimum temperature in the liquid decreases as pull speed increases as well. For the three pull speeds shown, the minimum liquid temperature, which occurs just upstream of the triple junction, was 1672, 1665, and $1660 K$ respectively. Conversely, the minimum temperature in the solid increases with pull speed with values of 1571,1601 , and $1627 \mathrm{~K}$. These changes have implications for the robustness of the growth process as removing heat through the liquid causes thermal instabilities [32].

Figure 10 shows the flow speed contours in the liquid for the same three pull speeds used to show the temperature contours. These pull speeds correspond to Peclet numbers, $\rho c u_{s, 1} d / k_{l}$, of $0.62,0.83$, and 1.1 so the temperature contours should mainly be determined by heat diffusion rather than the flow. However, the Marangoni stress on the liquid free surface causes there to be much larger velocities in the domain than the pull speed. For these conditions, the peak velocity, which occurred just upstream of the triple junction, was 10, 13, and $15 \mathrm{~cm} / \mathrm{s}$. This is roughly a factor of 10 larger than the pull speeds so convective effects are more significant than might be thought based on the pull speeds. The Marangoni stress pulls from hot to cold. Because the liquid becomes more supercooled approaching the interface, this causes a thin jet of fluid to 
flow along the free surface towards the triple junction. Approaching the triple junction the temperature passes through a minimum and then increases to the value at the triple junction. This causes a complex flow in the region near the triple junction where there is a counterclockwise rotating eddy situated in front of the facet as shown in the blow-up region in the figures. See [33] for a localized analysis of flow near a triple junction. The jet of fluid from the free surface passes under this vortex and then creates a clockwise vortex between the sheet and the bottom of the domain. Although difficult to see in the figure, this clockwise rotating vortex generates a chain of weaker alternating-rotation vortices both upstream and downstream. The Reynolds number of the flow based on the velocity of $10 \mathrm{~cm} / \mathrm{s}$ and the depth of $1.3 \mathrm{~cm}$ is 4700 so the flow itself is convectively dominated and nearly transitional.

\section{Stefan Solution}

To demonstrate the important role that kinetics have in predicting this problem, a comparison is made to simulations with no kinetics. Figure 11 shows temperature contours and the interface position from calculations of Case 3 with no kinetics at a pull speed of $1.3 \mathrm{~mm} / \mathrm{s}$ which is comparable to the pull speed used for the first plot of figure 7. For this case, it was difficult to obtain solutions with full-strength Marangoni effects. The calculation shown was

done with a value of $\frac{d \sigma}{d T}$ that was $10 \%$ of the value used for figure 7 . The main difference between the Stefan solution and the solution with kinetics is that a thin finger of solid forms on the top surface and extends towards the upstream edge of the cold block. This finger terminates at the location $x_{1} \approx-2 \mathrm{~cm}$. The blow-up region shows the tip of the solidification front. Also shown in the 
blow-up is that there is a very small region of supercooled fluid even in the Stefan solution. This phenomenon has been observed and discussed in [21].

Figure 12 shows the interface shape. As the heat removal profile upstream of the jet is fairly constant, the wedge behavior discussed in many previous papers can be seen where the vertical growth rate and horizontal pull rate balance to create a triangular shape. The angle of the wedge is approximately $2.2^{\circ}$. The heat flux upstream of jet is roughly constant around $43 \mathrm{~W} / \mathrm{cm}^{2}$. Dividing by $\rho L_{f}$ gives a growth velocity of $0.1 \mathrm{~mm} / \mathrm{s}$. For a pull speed of 1.3 $\mathrm{mm} / \mathrm{s}$ this corresponds to a wedge angle of $4.1^{\circ}$. The reason the actual growth

angle is smaller is that the liquid provides a net heat flux to the interface of around $25 \mathrm{~W} / \mathrm{cm}^{2}$, which reduces the solidification rate. Factoring this in gives a solidification velocity of $0.04 \mathrm{~mm} / \mathrm{s}$ and an estimated wedge angle of $1.8^{\circ}$ which bounds the observed wedge angle. Although not visible in the plot, the angle at which the interface meets the top surface at the triple junction is not equal to the wedge angle as there is curvature near the triple junction. By zooming in, this angle was estimated to be somewhere between $0.67^{\circ}$ and $1.9^{\circ}$ but was difficult to estimate because the curvature was on the scale of the grid resolution. The theory in [31, given by $\sin (2 \theta)=2 \Delta q /\left(\rho u_{s, 1} L_{f}\right)$ where $\Delta q$ is the jump in heat flux at the triple junction, predicts an angle of $1.6^{\circ}$. Thus, the Stefan solution is in good agreement with theory, but qualitatively different than what is observed in the experiment.

\section{Turning Point Behavior and Comparison to Experiment}

As mentioned in the introduction, the main point of comparison between the experiment and the simulations is the leading edge location as a function of 
pull speed. Figure 13 shows plots of triple junction location, $x_{t j}$, as a function of pull speed for the three cases listed in table 1. Experimental data for these cases are shown by $\times$ symbols and error bars are given for the experimental data points. There are two curves shown on each plot, the lower of the two was calculated without Marangoni effects and the upper included them. Obtaining converged steady solutions with Marangoni effects was difficult especially at higher pull speeds because of the increased supercooling and thus increased Marangoni velocities. Examining the curves for Case 1 reveals a common behavior seen in all of our calculations of this problem. As the pull speed increases, the location of the triple junction moves downstream until at some pull speed it passes under the center of the helium jet $\left(x_{t j} \geq 0\right)$. At a slightly higher pull speed there is a turning point in the solution. This turning point is a subcritical bifurcation and our Newton-Rhapson solver only was able to follow the stable branch towards the turning point. Near the turning point, it was difficult to obtain converged solutions, and we never were able to converge to a solution on the unstable branch. The implications of the turning point are that, unlike predictions of the wedge model, there is a pull speed limitation in horizontal ribbon growth process. The maximum pull speed attainable depends on the heat removal conditions as shown by the variation in the location of the turning point between the three cases. Case 3, which has the highest peak heat flux, had the highest turning point pull speed.

In all three cases, the numerical prediction and the experiment agreed to within $1 \mathrm{~mm}$ and the numerical simulations also well predicted the change in triple junction location with pull speed. There is some consistent error between the two results, which could come from many sources (thermal boundary conditions, kinetic constants, material properties, etc...) We have not determined 
the most likely cause for this difference, however, as shown below, this model is much more predictive than a Stefan model.

It was difficult to run the experiment at higher pull speeds because it became highly susceptible to perturbation; at pull speeds where the triple junction location was near the center of the jet, dendrites would form or an oscillatory behavior might set in. This is not surprising given that the amount of supercooling increases as the turning point is approached. In any case, the maximum pull speeds obtained in these validation experiments were around $2 \mathrm{~mm} / \mathrm{s}$, which is slightly lower than previously reported maximum pull speeds (discussed in the introduction). The actual maximum depends on the heat removal rate, but both the Kudo [10] and the Bates and Jewett [9] experiments report using a helium jet so the heat transfer rates are likely similar between all the experiments.

Figure 14 shows the triple junction position versus pull speed as calculated by the Stefan model. For the Stefan model, the triple junction stabilizes at a location that is at the edge of the cold block i.e. nearly $2 \mathrm{~cm}$ upstream of the jet. In addition, the Stefan model did not predict any upper limit to the pull speed. Stable pull speeds were predicted at $10 \mathrm{~cm} / \mathrm{s}$, which is an order of magnitude beyond any value ever attained in a horizontal ribbon growth experiment. The curve in figure 14 stops because at high pull speeds the sheet was so thin that it became difficult to resolve numerically. At the highest pull speed the sheet was $45 \mu \mathrm{m}$ thick. The fact that the triple junction location is completely different than that observed in the experiment and that the Stefan model predicts no limit to the pull speed confirms that solidification kinetics and faceted edge growth play an important role in the physics of horizontal ribbon growth. 


\section{Conclusions}

New experimental results obtained using the floating silicon method to produce single-crystal silicon have been presented that provide more insight into the process than previous experiments on horizontal ribbon growth. The data on the position of the triple junction as a function of pull speed can be used to quantitatively validate modeling approximations and to gain physical insight into the growth process.

Comparing Stefan model simulation results to the experimental results showed that the Stefan model did not correctly predict the location of the triple junction observed in the experiments nor did it predict the sensitivity of the triple

junction location to changes in pull speed. Almost all previous analyses of horizontal ribbon growth are based on the "wedge" model, which neglects kinetic effects. These results show that this model provides little useful insight into the physics of HRG.

Simulation results with kinetics showed that single-crystal horizontal ribbon growth is primarily a faceted growth phenomenon. The leading edge of the front is a $\{111\}$ facet where a step propagation mechanism of solidification is most likely active. In our further experimental investigations of FSM [25], we have more direct evidence for the presence of facets during the growth process, however there are still phenomena observed in the experiment that are not predicted by the model. This is discussed in more detail in [25].

The triple junction position predicted by this model agrees quantitatively with that observed in the experiment. Furthermore for a given heat removal rate, this model predicts a turning point in the triple junction versus pull speed 
response which is consistent with all previous experimental observations of the existence of a maximum attainable pull speed in HRG of single-crystal silicon. This model also showed that increased pull speeds lead to increased supercooling, which is consistent with experimental observations of transition to dendritic growth at high pull speeds.

\section{Acknowledgement}

This material is based upon work supported, in part, by the Department of Energy under grant DE-EE0000595. Thanks to Applied Materials, Inc., Varian Semiconductor Equipment Division (VSED), for supplying the funding and motivation for this work.

\section{References}

[1] Ciszek T. Techniques for the crystal growth of silicon ingots and ribbons. Journal of Crystal Growth 1984; 66(3):655-672.

[2] Antonov P, Kurlov V. A review of developments in shaped crystal growth of sapphire by the Stepanov and related techniques. Progress in crystal growth and characterization of materials 2002; $\mathbf{4 4}(2): 63$.

[3] Ciszek T. Photovoltaic silicon crystal growth. Bulk Crystal Growth of Electronic, Optical $\&$ Optoelectronic Materials 2005; :451-476.

[4] Narayanan M, Ciszek T. Silicon solar cells: Materials, devices, and manufacturing. Springer Handbook of Crystal Growth 2010; :1701-1718.

[5] Falckenberg R, Grabmaier J, Hediger F. Finite element analysis of hor- 
izontal silicon sheet growth from the melt. Journal of Crystal Growth 1987; 82(1):10-16.

[6] Steinbach I, Hofs H. Microstructural analysis of the crystallization of silicon ribbons produced by the RGS process. Photovoltaic Specialists Conference, 1997., Conference Record of the Twenty-Sixth IEEE, IEEE, 1997; 91-93.

[7] Shockley W. Process for growing single crystals. http://www.google.com/patents/US3031275 April 1962. US Patent $3,031,275$.

[8] Bleil C. A new method for growing crystal ribbons. Journal of Crystal Growth 1969; 5(2):99-104.

[9] Bates H, Jewett D. Low angle silicon sheet growth: A review of progress, problems and promise. Flat-Plate Solar Array Proj. Res. Forum on the High-Speed Growth and Characterization of Crystals for Solar Cells, vol. 1, 1984; 297-307.

[10] Kudo B. Improvements in the horizontal ribbon growth technique for single crystal silicon. Journal of Crystal Growth 1980; 50(1):247-259.

[11] Cheng Y. The growth of dislocation-free bicrystal silicon ribbon. Journal of Physics D: Applied Physics 1993; 26:1109.

[12] Cheng Y. The fractal structure in dislocation-free bicrystal silicon ribbon. Journal of Physics D: Applied Physics 1994; 27:1938.

[13] Hoyler G, Falckenberg R, Grabmaier J, Freienstein B. Fast Si sheet growth by the horizontal supported web technique. Journal of Crystal Growth 1986; 79(1-3):572-577.

[14] Bleil C. A new approach to continuous crystal sheet growth. Journal of Crystal Growth 1990; 104(1):29-33.

[15] Zoutendyk J. Theoretical analysis of heat flow in horizontal ribbon growth 
from a melt. Journal of Applied Physics 1978; 49(7):3927-3932.

[16] Zoutendyk J. Analysis of forced convection heat flow effects in horizontal ribbon growth from the melt. Journal of Crystal Growth 1980; 50(1):8393.

[17] Glicksman M, Voorhees P. Analysis of morphologically stable horizontal ribbon crystal growth. Journal of Electronic Materials 1983; 12(1):161179.

[18] Thomas P, Brown R. Rate limits in silicon sheet growth: The connections between vertical and horizontal methods. Journal of Crystal Growth 1987; 82(1):1-9.

[19] Ranjan S, Balaji S, Panella R, Ydstie B. Silicon solar cell production. Computers $\&$ Chemical Engineering 2011; 35:1439-1453.

[20] Daggolu P, Yeckel A, Bleil CE, Derby JJ. Thermal-capillary analysis of the horizontal ribbon growth of silicon crystals. Journal of Crystal Growth 2012; 355(1):129-139.

[21] Daggolu P, Yeckel A, Bleil CE, Derby JJ. Stability limits for the horizontal ribbon growth of silicon crystals. Journal of Crystal Growth 2013; 363:132-140.

[22] Oliveros GA, Sridhar S, Ydstie BE. Existence and static stability of the meniscus in horizontal ribbon growth. Journal of Crystal Growth 2015; 411:96-105.

[23] Oliveros GA, Liu R, Sridhar S, Ydstie BE. Silicon wafers for solar cells by horizontal ribbon growth. Industrial \& Engineering Chemistry Research 2013; 52(9):3239-3246.

[24] Daggolu P, Yeckel A, Derby JJ. An analysis of segregation during horizontal ribbon growth of silicon. Journal of Crystal Growth 2014; 390:80-87.

[25] Kellerman P, Kernan B, Helenbrook BT, Sun D, Sinclair F, Carlson F. 
Floating silicon method single crystal ribbon-observations and proposed limit cycle theory. Journal of Crystal Growth 2016; Submitted.

[26] Ciszek T. Crystallographic growth forms of silicon on a free melt surface. Journal of The Electrochemical Society 1985; 132(2):422-427.

[27] Mito M, Tsukada T, Hozawa M, Yokoyama C, Li YR, Imaishi N. Sensitivity analyses of the thermophysical properties of silicon melt and crystal. Measurement Science and Technology 2005; 16(2):457.

[28] Weinstein O, Brandon S. Dynamics of partially faceted melt/crystal interfaces I: computational approach and single step-source calculations. Journal of Crystal Growth 2004; 268(1):299-319.

[29] Howel JR, Siegel R, Mengü MP. Thermal radiation heat transfer. 5th edn., CRC press, 2001. URL http://www.thermalradiation.net/sectionb/ B-71.html.

[30] Helenbrook BT. Solidification along a wall or free surface with heat removal. Journal of Crystal Growth 2015; 418:79 - 85, doi:http://dx.doi. org/10.1016/j.jcrysgro.2015.02.028. URL http://www.sciencedirect. com/science/article/pii/S0022024815001189.

[31] Helenbrook BT. High-order adaptive arbitrary-Lagrangian-Eulerian (ALE) simulations of solidification. Int. J. Num. Meth. Eng. 2016; Under revision.

[32] Mullins WW, Sekerka RF. Stability of a planar interface during solidification of a dilute binary alloy. Journal of Applied Physics 1964; 35(2):444451, doi:10.1063/1.1713333. URL http://link.aip.org/link/?JAP/ $35 / 444 / 1$.

[33] Anderson D, Davis S. Local fluid and heat flow near contact lines. Journal of Fluid Mechanics 1994; 268:231-266. 


\section{List of Figures}

$1 \quad$ Schematic of the experimental configuration. 33

$2 \quad$ Typical silicon sheet produced by HRG. Top view. 34

$3 \quad$ Side view of sheet pulled slowly from the furnace. Growth direction is to the left. $\{111\}$ facet is observed at the leading edge.

$4 \quad$ Schematic showing the physical domain, spatial coordinates, and the initial mesh for the solidification simulations.

$5 \quad$ Heat flux profiles for three different experimental configurations. Dashed lines are curve fits.

$6 \quad$ Heat flux into bottom of domain. Solid line is from Fluent simulation of entire furnace. Dashed line is a curve fit. 38

7 Temperature contours for Case 3 at three different pull speeds: $1.2,1.6$, and $2.2 \mathrm{~mm} / \mathrm{s}$. Temperature contours correspond to $5 K$ intervals from 1665 to $1710 K$ with two additional contours at $T_{m} \pm 2.5 \mathrm{~K}$ where $T_{m}$ is $1685 \mathrm{~K}$. The two contours shown in the liquid in the blow region are $T_{m}-2.5 K$ and $T_{m}-5 K$.

8 Thickness of the sheet as a function of pull speed for Case 3 (solid line). Dashed line shows an inversely proportional relationship.

$9 \quad$ Misalignment angle as a function of position for Case 3 with a pull speed of $2.2 \mathrm{~mm} / \mathrm{s}$. Faceted region corresponds to $\theta>-1$ which has a horizontal length of $60 \mu \mathrm{m}$. Inset shows the shape of the interface with labels $\mathrm{A}, \mathrm{B}$, and $\mathrm{C}$ on both plots corresponding to the triple junction, the beginning of the elbow, and the end of the elbow respectively.

10 Flow speed contours for Case 3 at three different pull speeds: $1.2,1.6$, and $2.2 \mathrm{~mm} / \mathrm{s}$. Legend shows magnitudes in $\mathrm{cm} / \mathrm{s}$. 42

11 Temperature contours for Case 3 with no kinetics at a pull speed of $1.3 \mathrm{~mm} / \mathrm{s}$. Temperature contours correspond to $5 \mathrm{~K}$ intervals from 1665 to $1710 K$ with two additional contours at $T_{m} \pm 2.5 \mathrm{~K}$ where $T_{m}$ is $1685 \mathrm{~K}$. 
12 Interface shape for Case 3 with no kinetics at a pull speed of $1.3 \mathrm{~mm} / \mathrm{s}$.

13 Triple junction position as a function of pull speed for Cases 1, 2, and 3. Upper curve includes Marangoni effect. $\times$ symbol indicates experimental data. Dashed line at $x_{t j}=0$ indicates the center of the helium jet.

14 Triple junction position as a function of pull speed as calculated by the Stefan model. 


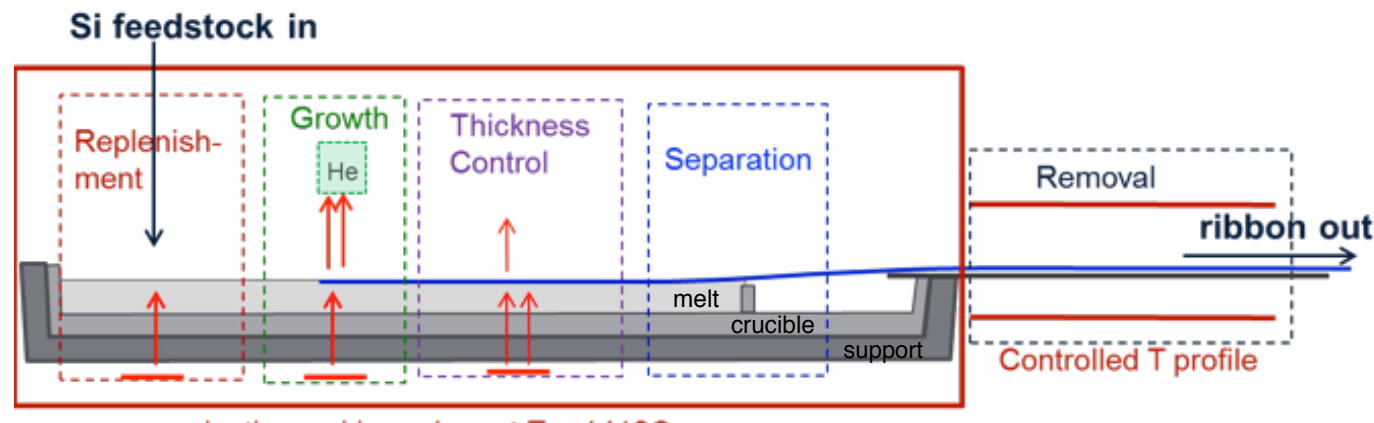

Isothermal boundary at $T_{m}=1412 \mathrm{C}$

Fig. 1. Schematic of the experimental configuration. 


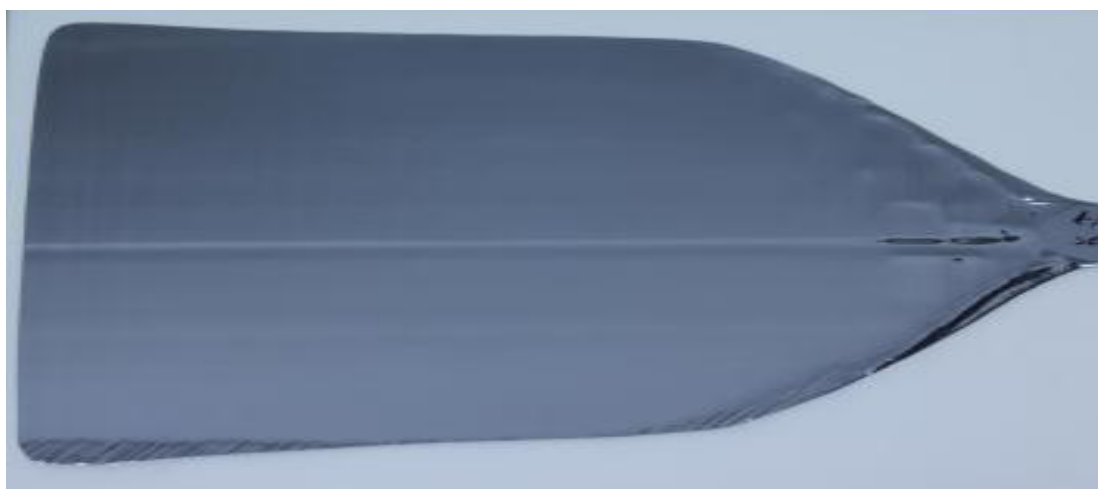

Fig. 2. Typical silicon sheet produced by HRG. Top view. 


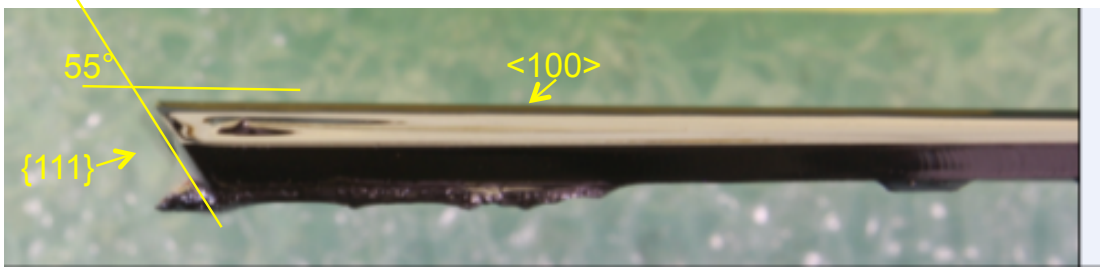

Fig. 3. Side view of sheet pulled slowly from the furnace. Growth direction is to the left. $\{111\}$ facet is observed at the leading edge. 


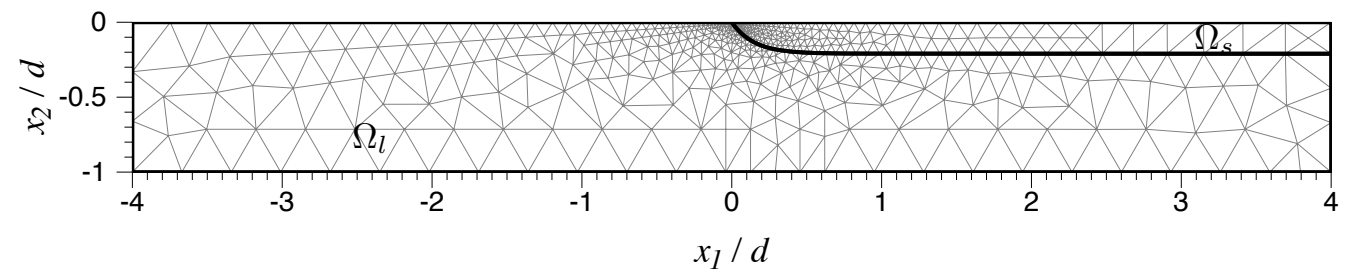

Fig. 4. Schematic showing the physical domain, spatial coordinates, and the initial mesh for the solidification simulations. 


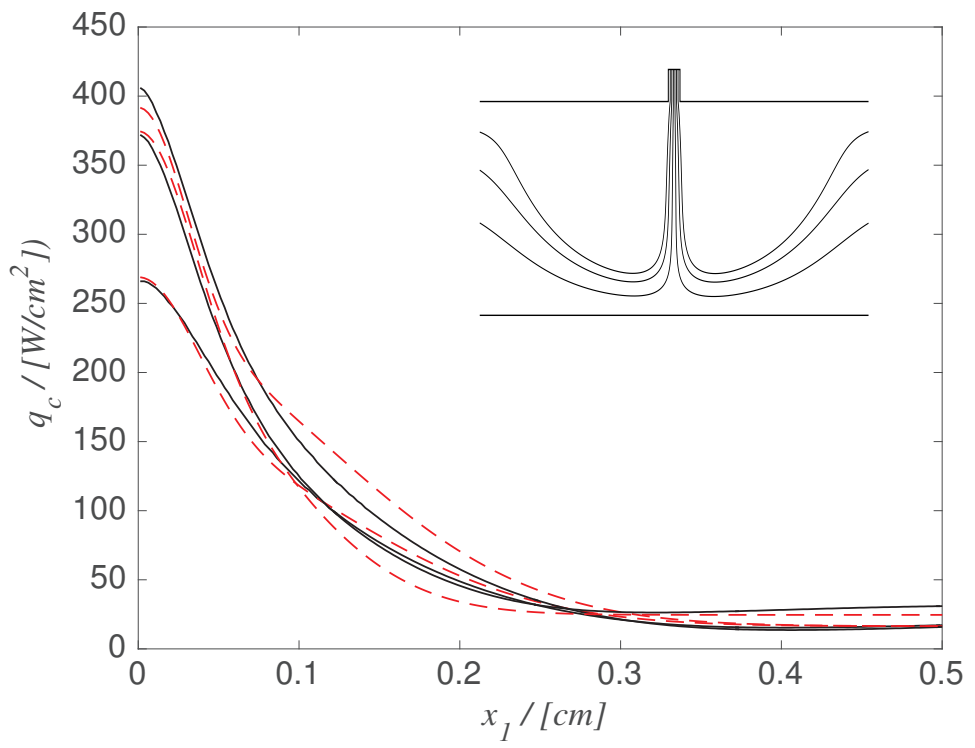

Fig. 5. Heat flux profiles for three different experimental configurations. Dashed lines are curve fits. 


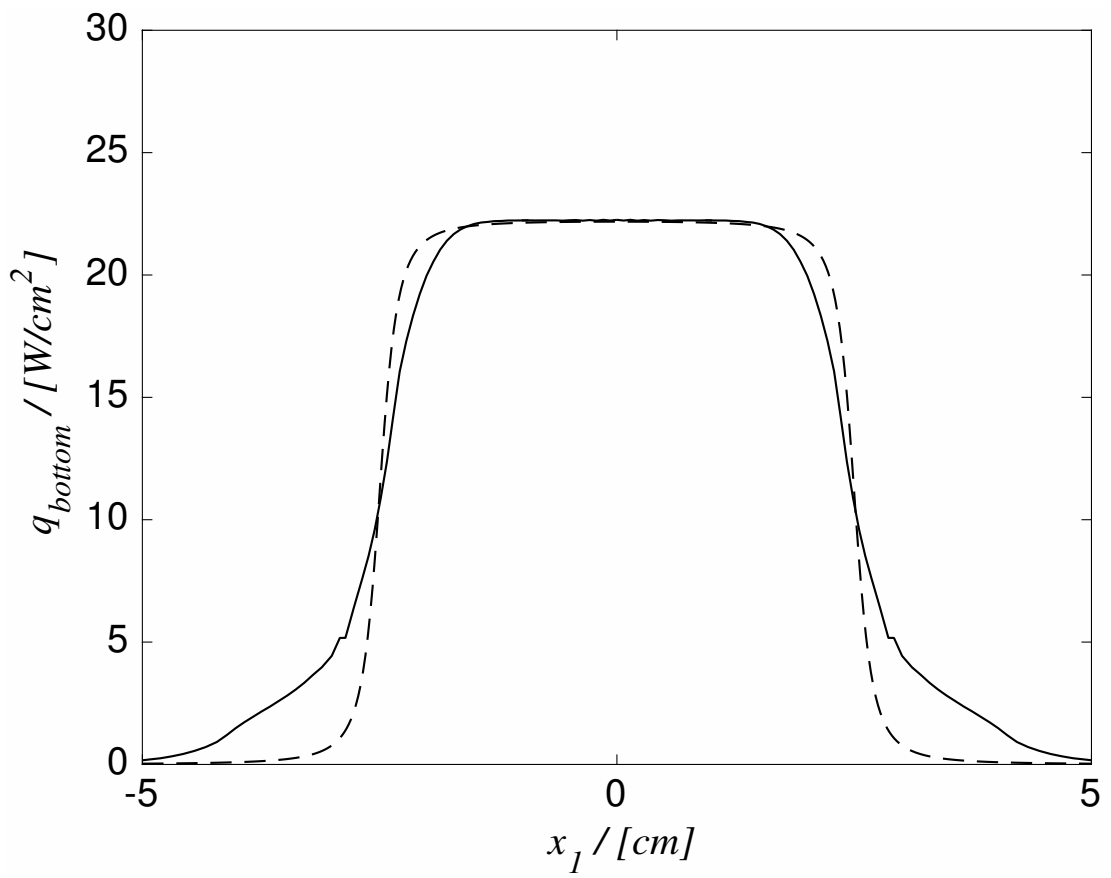

Fig. 6. Heat flux into bottom of domain. Solid line is from Fluent simulation of entire furnace. Dashed line is a curve fit. 

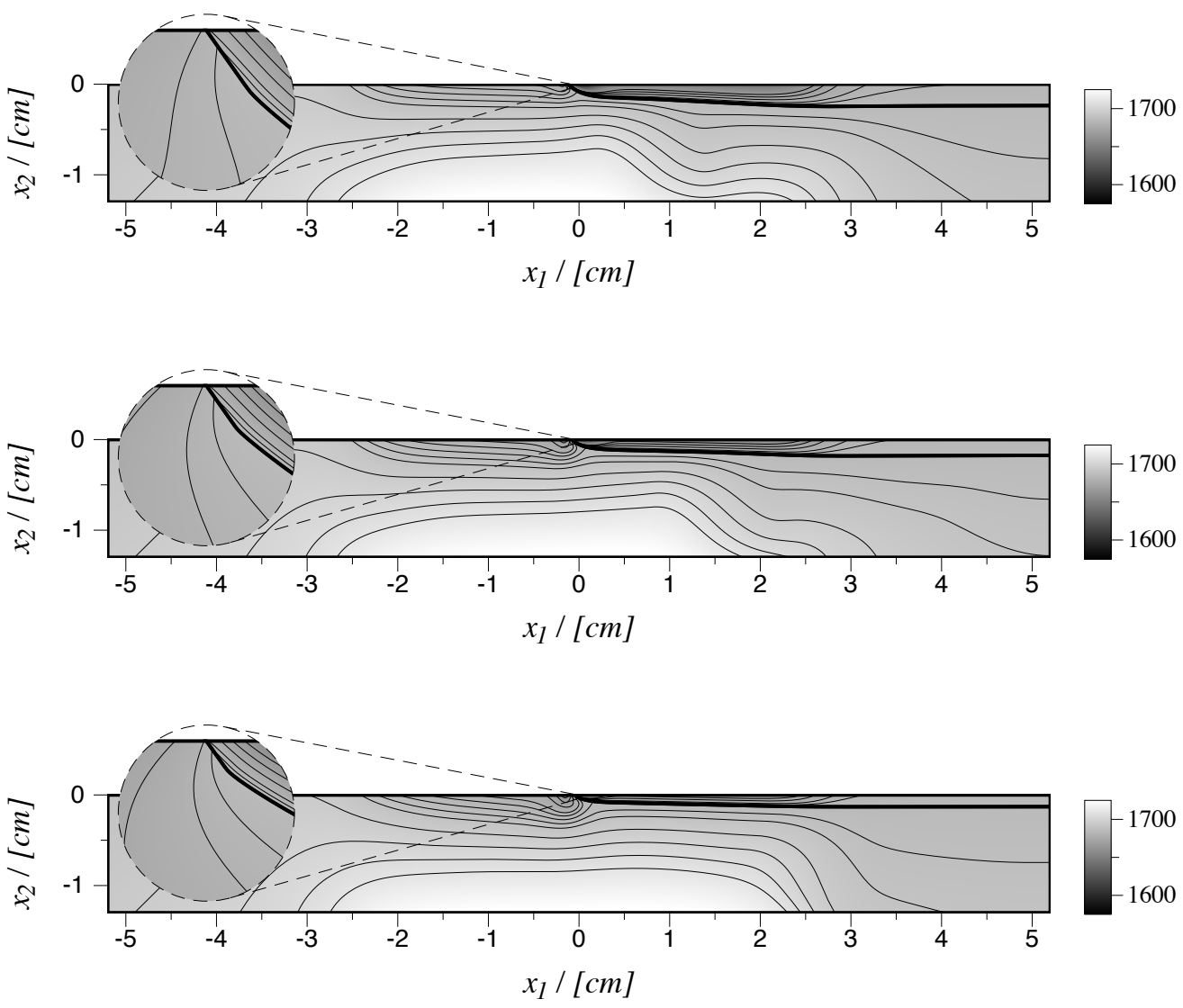

Fig. 7. Temperature contours for Case 3 at three different pull speeds: 1.2, 1.6, and $2.2 \mathrm{~mm} / \mathrm{s}$. Temperature contours correspond to $5 \mathrm{~K}$ intervals from 1665 to $1710 \mathrm{~K}$ with two additional contours at $T_{m} \pm 2.5 \mathrm{~K}$ where $T_{m}$ is $1685 \mathrm{~K}$. The two contours shown in the liquid in the blow region are $T_{m}-2.5 \mathrm{~K}$ and $T_{m}-5 \mathrm{~K}$. 


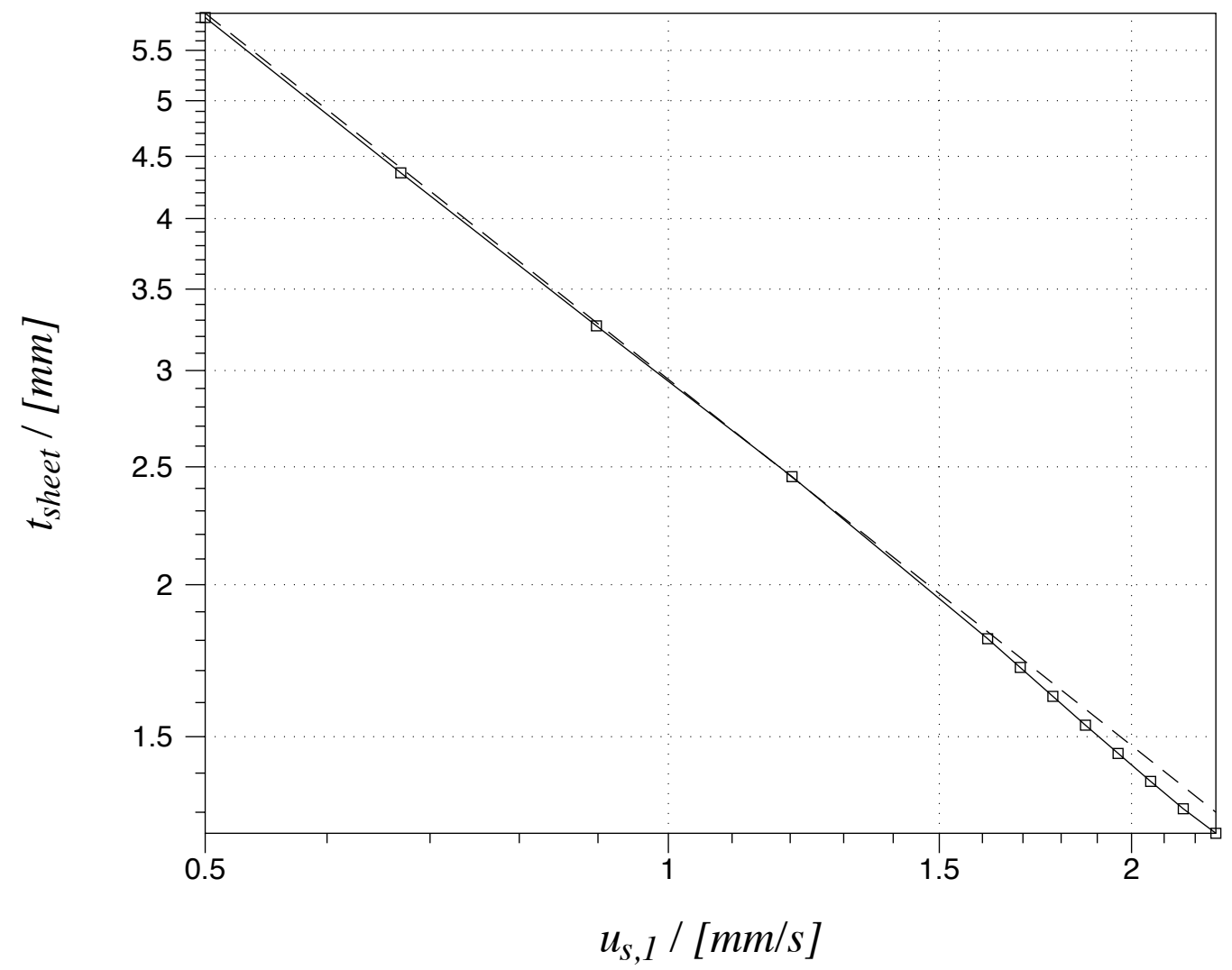

Fig. 8. Thickness of the sheet as a function of pull speed for Case 3 (solid line). Dashed line shows an inversely proportional relationship. 


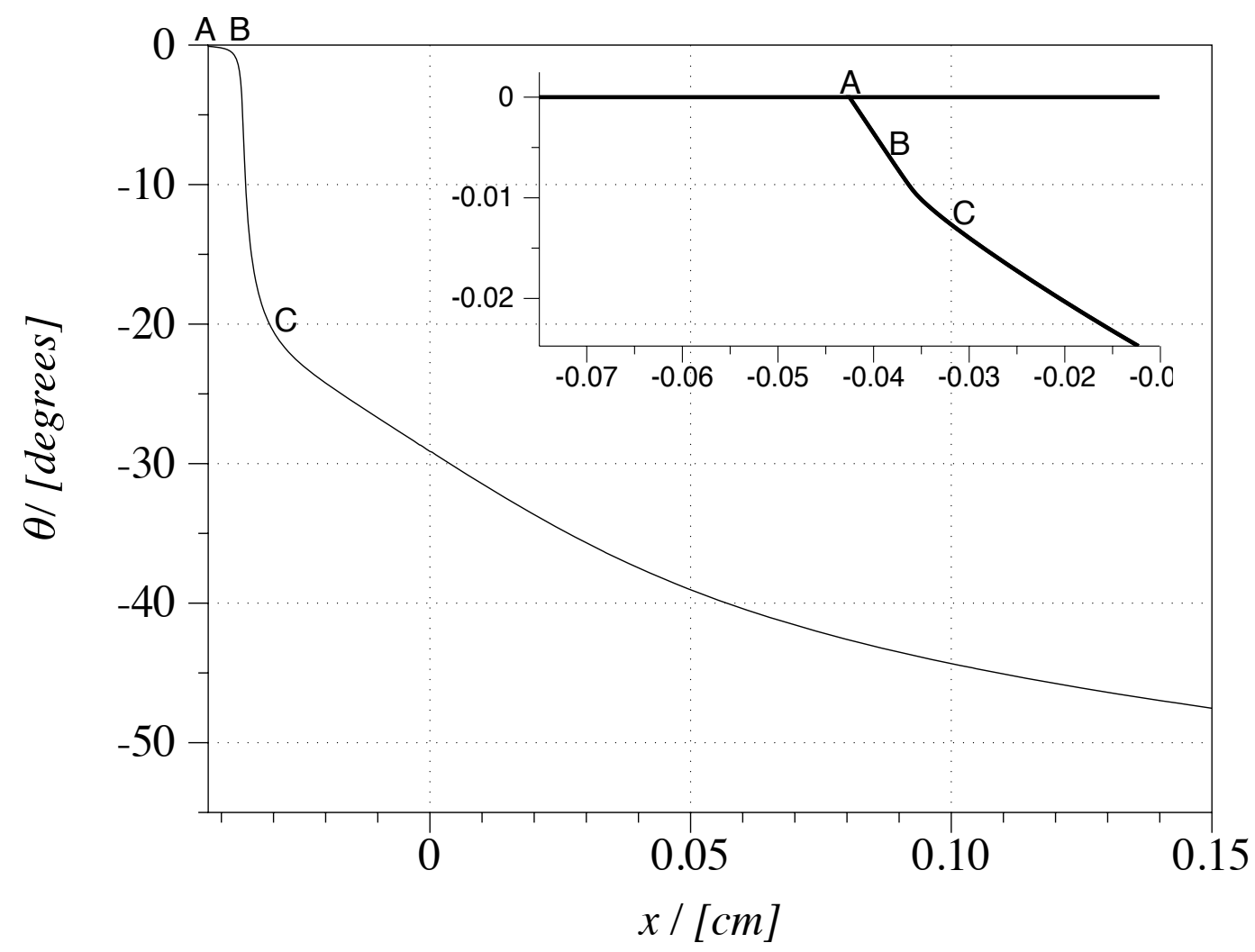

Fig. 9. Misalignment angle as a function of position for Case 3 with a pull speed of $2.2 \mathrm{~mm} / \mathrm{s}$. Faceted region corresponds to $\theta>-1$ which has a horizontal length of $60 \mu \mathrm{m}$. Inset shows the shape of the interface with labels A, B, and C on both plots corresponding to the triple junction, the beginning of the elbow, and the end of the elbow respectively. 

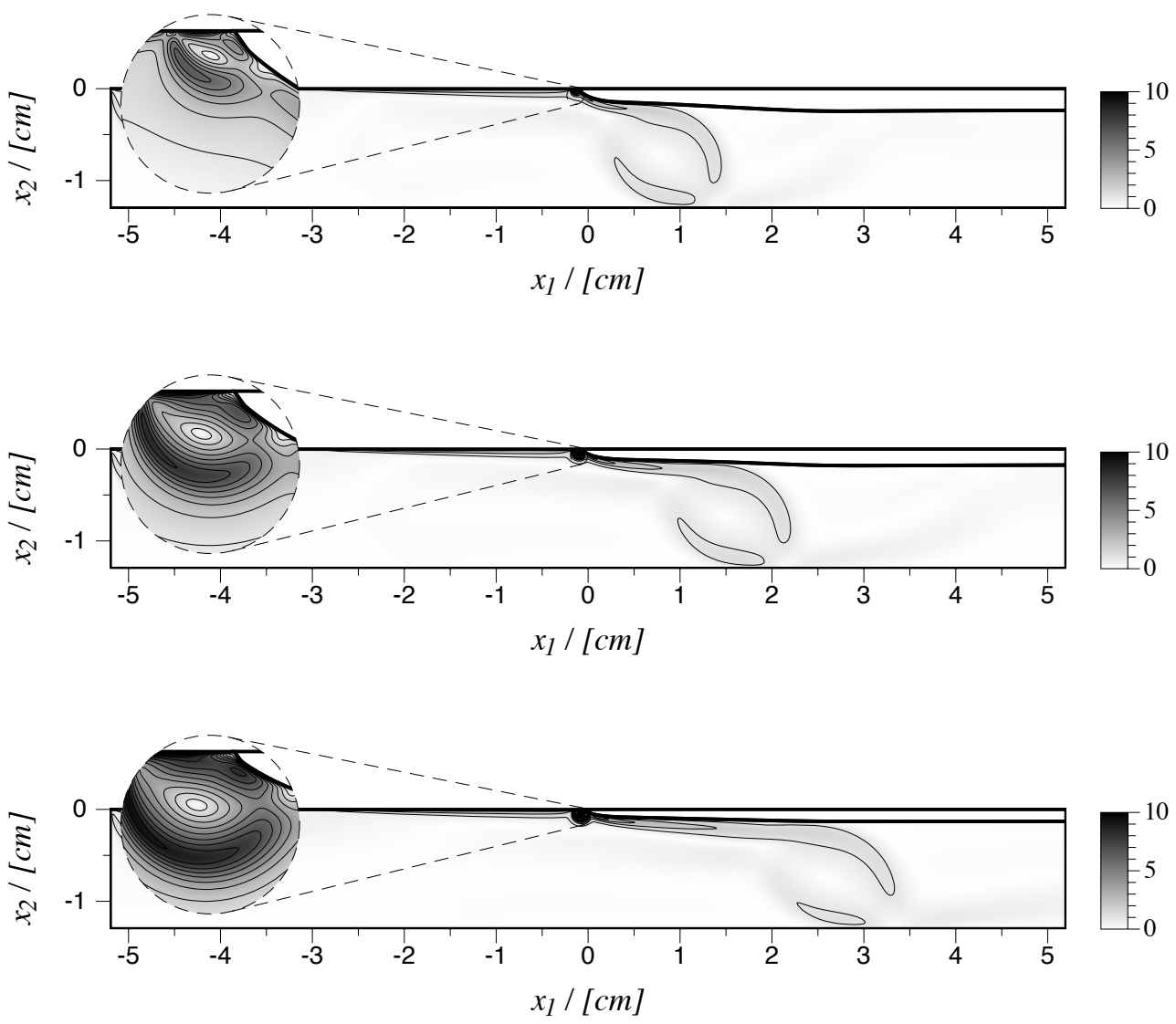

Fig. 10. Flow speed contours for Case 3 at three different pull speeds: 1.2, 1.6, and $2.2 \mathrm{~mm} / \mathrm{s}$. Legend shows magnitudes in $\mathrm{cm} / \mathrm{s}$. 


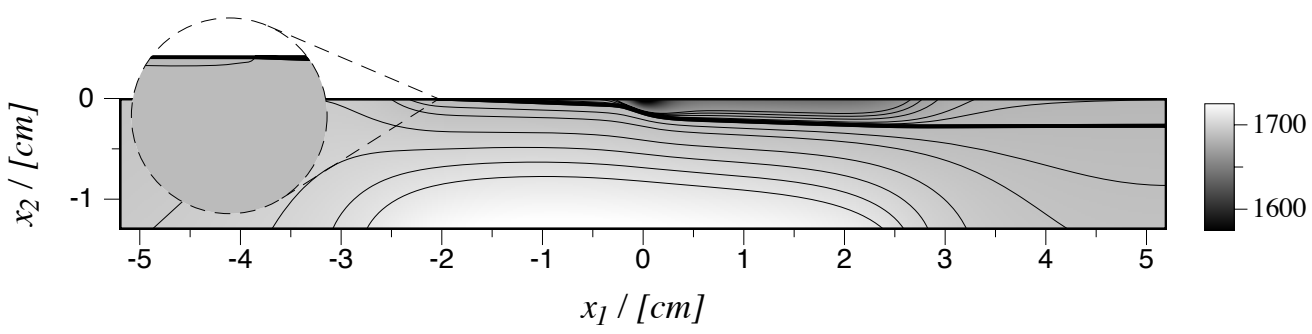

Fig. 11. Temperature contours for Case 3 with no kinetics at a pull speed of 1.3 $\mathrm{mm} / \mathrm{s}$. Temperature contours correspond to $5 \mathrm{~K}$ intervals from 1665 to $1710 \mathrm{~K}$ with two additional contours at $T_{m} \pm 2.5 \mathrm{~K}$ where $T_{m}$ is $1685 \mathrm{~K}$. 


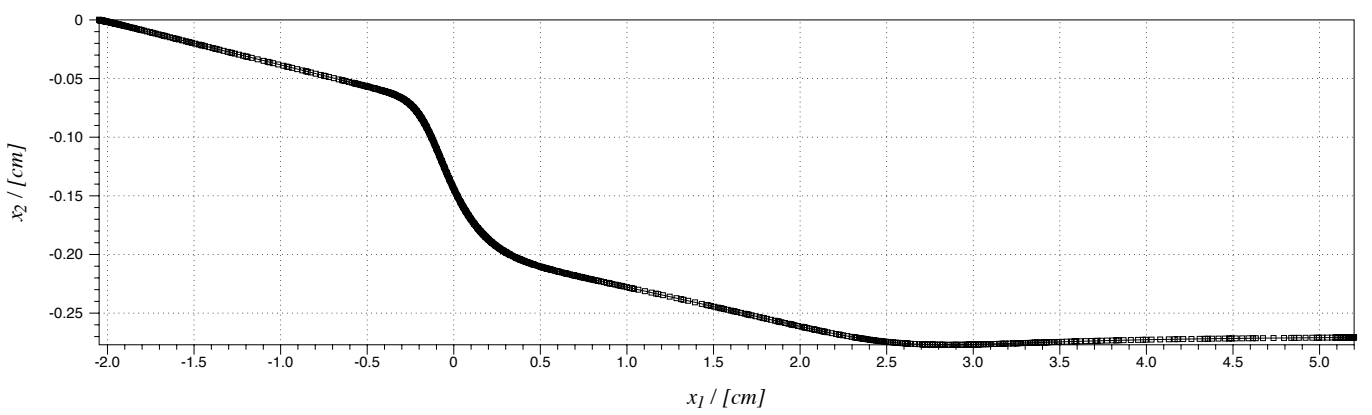

Fig. 12. Interface shape for Case 3 with no kinetics at a pull speed of $1.3 \mathrm{~mm} / \mathrm{s}$. 

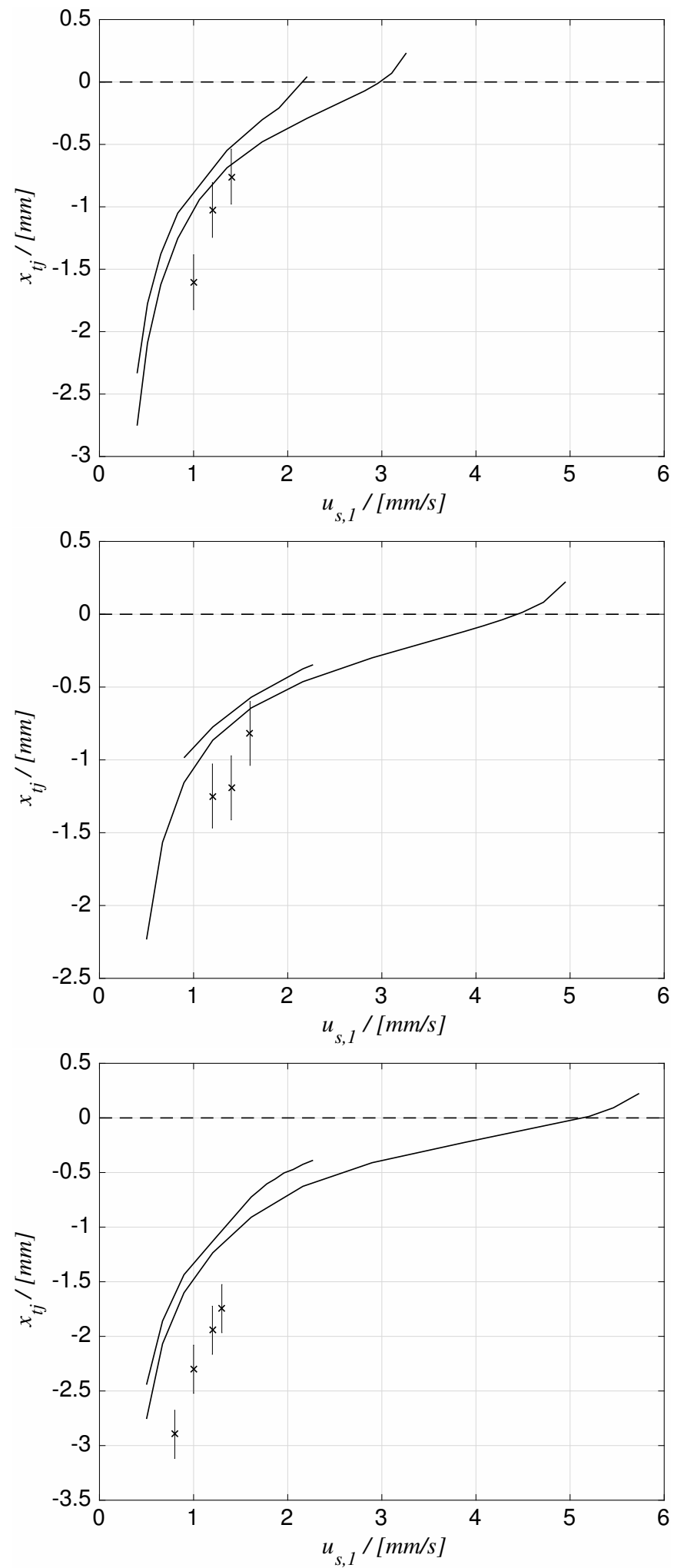

Fig. 13. Triple junction position as a function of pull speed for Cases 1, 2, and 3. Upper curve includes Marangoni effect. $\times$ symbol indicates experimental data. Dashed line at $x_{t j}=0$ indicates the center of the helium jet. 


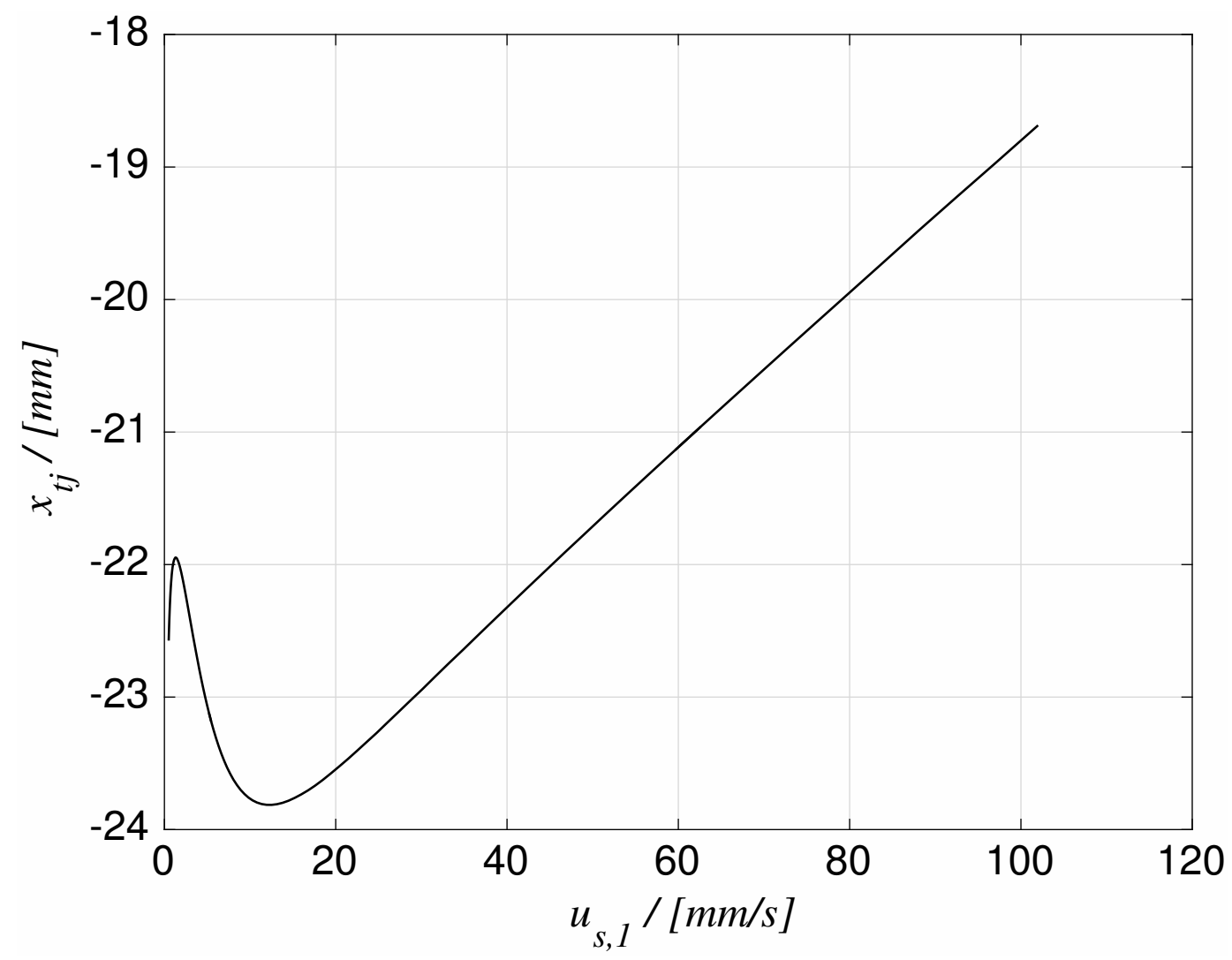

Fig. 14. Triple junction position as a function of pull speed as calculated by the Stefan model. 


\section{List of Tables}

$1 \quad$ Helium jet curve fit parameters used model growth experiments 48 


\begin{tabular}{cccccc}
\hline Case \# & $\begin{array}{c}w \\
{[\mathrm{~mm}]}\end{array}$ & $\begin{array}{c}w_{b} \\
{[\mathrm{~mm}]}\end{array}$ & $\theta$ & $\begin{array}{c}q_{\text {base }} \\
{\left[W / \mathrm{cm}^{2}\right]}\end{array}$ & $\begin{array}{c}q_{\text {peak }} \\
{\left[W / \mathrm{cm}^{2}\right]}\end{array}$ \\
\hline 1 & 0.42 & 1.44 & 0.55 & 16.4 & 253 \\
2 & 0.38 & 0.96 & 0.55 & 24.6 & 350 \\
3 & 0.34 & 1.44 & 0.55 & 16.4 & 375 \\
\hline
\end{tabular}

Table 1

Helium jet curve fit parameters used to model growth experiments 